\title{
Relation flore potentielle-flore réelle de sols agricoles de Côte-d'Or
}

\author{
G Barralis *, F Dessaint, R Chadœuf \\ Laboratoire de malherbologie, Inra, BV 1540, F-21034 Dijon cedex, France
}

(Reçu le 9 mai 1996; accepté le 28 août 1996)

\begin{abstract}
Résumé - L'estimation du stock semencier au semis de cultures d'automne et le dénombrement des levées au cours du cycle cultural ont été réalisés dans 35 parcelles agricoles de la plaine de Dijon (Côte-d'Or). La valeur médiane du stock semencier viable est estimée à 2864 semences par mètre carré, et le taux moyen de levée à $5,54 \%$ du nombre de semences viables. Ce taux de levée varie en fonction des espèces de $0,9 \%$ chez Kickxia spuria à $32,8 \%$ chez Polygonum persicaria. La dynamique des levées est influencée par les conditions climatiques, et un hiver froid et prolongé favorise des levées de printemps au détriment des levées d'automne pour les espèces à germination automnale préférentielle.
\end{abstract}

mauvaise herbe / stock semencier / levée / viabilité

Summary - Relationship between the seedbank and the actual weed flora in agricultural soils in the Côted'Or. The weed seedbank was estimated at the time of autumn crop sowing and the emergence of seedlings was counted during the cultural cycle in 35 fields on the Dijon plain (Côte-d'Or). The median value for the viable seedbank was estimated at 2864 seeds $/ \mathrm{m}^{2}$ and the average rate of emergence at $5.54 \%$ of viable seed content. This average rate varies from $0.9 \%$ for Kickxia spuria to $32.8 \%$ for Polygonum persicaria. The dynamics of seedlings emergence was influenced by the climatic conditions, and cold and prolonged winter favoured the emergence of spring seedlings to the detriment of the autumn seedlings for species with preferential autumn germinating.

weed / seedbank / seedling emergence / viability

\section{INTRODUCTION}

L'analyse du stock semencier des sols agricoles, reflet du passé cultural parcellaire, réalisée dans différentes conditions géographiques et agronomiques montre que ces sols renferment une quantité importante de semences viables (Kropac, 1966 ; Jensen, 1969 ; Roberts, 1981), même dans les régions où le désherbage chimique est régulièrement pratiqué depuis de nombreuses années (Roberts et Chancellor, 1986 ; Barralis et Chadœuf, 1987 ; Zanin et al, 1992).

L'estimation spécifique et quantitative de ce potentiel semencier peut permettre de prévoir les risques de salissement des cultures dont la végétation adventice au cours du cycle cultural ne

* Correspondance et tirés à part 
représente qu'une faible proportion de la flore potentielle. La plupart des auteurs considèrent le taux de levée au champ comme globalement inférieur à $10 \%$ (Barralis et Salin, 1973 ; Roberts et Ricketts, 1979 ; Roberts, 1981 ; Beuret, 1984 ; Roberts, 1984 ; Lambelet-Haueter, 1986 ; Barralis et Chadœuf, 1988 ; Debaeke, 1988b ; Ball et Miller, 1989 ; Wilson et Lawson, 1992 ; Wilson et Aebischer, 1995), ce taux ayant tendance à être plus élevé chez les graminées que chez les dicotylédones (Forcella et al, 1992).

La connaissance des relations spécifiques entre le stock semencier et les levées au champ est, de ce fait, un élément important pour la prévision des risques d'infestation d'une culture et pour la modélisation du cycle végétatif d'une mauvaise herbe et, à plus long terme, pour l'estimation des risques d'évolution de la flore.

L'objectif de ce travail est d'analyser les relations entre ces deux paramètres, stock semencier et levées, dans des cultures d'automne au cours de quatre campagnes de relevés au champ.

\section{MATÉRIEL ET MÉTHODES}

L'étude a été faite de 1986 (campagne 1986/1987) à 1990 (campagne 1990/1991) sur un ensemble de 35 parcelles agricoles du département de Côte-d'Or, toutes ensemencées à l'automne (cinq en colza, deux en orge, vingt-huit en blé). Notre étude portant sur la flore des cultures d'automne, les parcelles retenues sont généralement différentes à chaque campagne. L'analyse du stock semencier et le suivi de la flore de surface ont été réalisés dans une zone d'environ 1000 $\mathrm{m}^{2}$ située à plus de $20 \mathrm{~m}$ de la bordure du champ.

\section{Stock semencier}

Dans chaque parcelle, dès le semis de la culture, 90 prélèvements de sol ont été faits à l'aide d'une sonde de $30 \mathrm{~cm}$ de hauteur et $4,6 \mathrm{~cm}$ de diamètre. La hauteur réelle des carottes de terre se situe entre 14 et $25 \mathrm{~cm}$, avec une valeur médiane de $19,5 \mathrm{~cm}$. Ces prélèvements ont été regroupés pour être analysés selon la méthodologie déjà décrite (Barralis et Chadœuf, 1980) : la suspension aqueuse de sol est tamisée et la fraction comprise entre les tamis de maille 4 et $0,2 \mathrm{~mm}$, contenant les semences adventices est mise à germer en conditions contrôlées $\left(12\right.$ heures à $15^{\circ} \mathrm{C}$ sans éclairage et 12 heures à $25^{\circ} \mathrm{C}$ avec éclairage) pendant 1 mois. Ensuite, après dessiccation de la fraction terreuse, les semences bien constituées et qui n'ont pas germé sont dénombrées sous loupe binoculaire, puis à nouveau mises à germer en présence d'un agent de levée de dormance $\left(\mathrm{GA}_{3}\right.$ ou $\left.\mathrm{KNO}_{3}\right)$ dans les mêmes conditions contrôlées. L'ensemble de ces opé- rations permet pour chaque espèce de définir le nombre total de semences intactes (dénombrement total) et le nombre total de semences aptes à germer dans nos conditions expérimentales (par simplification nous l'appellerons dénombrement viable).

\section{Flore de surface}

Elle est régulièrement suivie du semis à la récolte de la plante cultivée dans 17 placettes de $12 \times 50 \mathrm{~cm}$ (représentant $1 \mathrm{~m}^{2}$ ) par parcelle matérialisées entre les lignes de semis dès la levée de la culture et protégées par des écrans plastiques lors des traitements herbicides. À chaque observation, la fréquence étant en moyenne de 15 jours, les jeunes plantes sont identifiées et délicatement éliminées. Cette technique permet de recenser l'ensemble des levées tout au long du cycle végétatif de la culture et donne des résultats comparables à une cartographie des levées (données non publiées).

\section{RÉSULTATS}

\section{Stock semencier et levées au champ}

Le stock semencier total des 35 parcelles varie de 546 à 23889 (médiane 4 682) et le stock semencier viable de 446 à 19793 (médiane 2 864) semences par $m^{2}$. Comme paramètre de tendance centrale nous avons préféré, chaque fois que l'effectif le permettait, utiliser la médiane moins sensible aux valeurs extrêmes que la moyenne. La viabilité (aptitude à la germination) des semences, toutes espèces confondues varie de 28,7 à 95,2 \% (médiane 70,8) (tableau I) avec néanmoins un coefficient de corrélation entre dénombrement viable et dénombrement total de 0,97 .

Ces résultats sont à rapprocher de ceux que nous avions obtenus antérieurement dans cette région agricole sur 50 parcelles échantillonnées : le stock total variait de 400 à 86526 (médiane 5 100) et le stock viable de 93 à 85301 (médiane 3 375) (Barralis et Chadceuf, 1987). Les valeurs médianes sont proches.

Les levées cumulées au cours du cycle végétatif de la culture varient de 8 à 1569 plantes par mètre carré (médiane 140).

\section{Relations stock-levées parcellaires}

Toutes espèces, parcelles et années confondues, les levées ne représentent que 5,5\% du 
Tableau I. Données générales parcellaires : culture et année de semis, levées au champ, dénombrement réel total et viable exprimés en nombre d'individus par mètre carré, rapports calculés levées/dénombrements et viabilité moyenne.

\begin{tabular}{|c|c|c|c|c|c|c|c|}
\hline Parcelle & Cultann & Lev & $D T$ & $D V$ & $L / D T$ & $L / D V$ & $D V / D T$ \\
\hline Corcelles 1 & $\mathrm{BH} 86$ & 635 & 10816 & 7726 & 5,9 & 8,2 & 71,4 \\
\hline Corcelles 2 & $\mathrm{BH} 86$ & 308 & 2957 & 2444 & 10,4 & 12,6 & 82,7 \\
\hline Fauvernay 1 & $\mathrm{BH} 86$ & 125 & 1752 & 899 & 7,1 & 13,8 & 51,3 \\
\hline Labergement 1 & $\mathrm{BH} 86$ & 241 & 4555 & 4236 & 5,3 & 5,7 & 93,0 \\
\hline Labergement 2 & $\mathrm{OH} 86$ & 140 & 1738 & 500 & 8,0 & 27,9 & 28,7 \\
\hline Total 1986 & & 1447 & 21818 & 15804 & 6,6 & 9,2 & 72,4 \\
\hline Belleneuve 1 & $\mathrm{BH} 87$ & 62 & 1672 & 666 & 3,7 & 9,3 & 39,8 \\
\hline Corcelles 3 & $\mathrm{BH} 87$ & 122 & 4049 & 2491 & 3,0 & 4,9 & 61,5 \\
\hline Corcelles 4 & $\mathrm{CO} 87$ & 110 & 2204 & 1239 & 5,0 & 8,8 & 56,2 \\
\hline Fauvernay 2 & CO 87 & 289 & 5681 & 3923 & 5,1 & 7,4 & 69,1 \\
\hline Fauvernay 3 & CO 87 & 289 & 5681 & 3923 & 5,1 & 7,4 & 69,1 \\
\hline Labergement 1 & BM 87 & 100 & 7686 & 7000 & 1,3 & 1,4 & 97,2 \\
\hline Labergement 2 & CO 87 & 84 & 1792 & 1159 & 4,7 & 7,2 & 64,7 \\
\hline Labergement 3 & CO 87 & 145 & 10070 & 9011 & 1,4 & 1,6 & 89,5 \\
\hline Longecourt 1 & $\mathrm{BH} 87$ & 8 & 546 & 446 & 1,5 & 1,8 & 81,7 \\
\hline Magny 1 & $\mathrm{BH} 87$ & 191 & 1638 & 1126 & 11,7 & 17,0 & 68,7 \\
\hline Total 1987 & & 1186 & 39381 & 29890 & 3,0 & 4,0 & 75,9 \\
\hline Corcelles 3 & $\mathrm{BH} 88$ & 276 & 7120 & 6520 & 3,9 & 4,2 & 91,6 \\
\hline Corcelles 4 & $\mathrm{BH} 88$ & 127 & 1132 & 659 & 11,2 & 19,3 & 58,2 \\
\hline Longvic 1 & $\mathrm{BH} 88$ & 389 & 12614 & 9843 & 3,1 & 4,0 & 78,0 \\
\hline Longvic 2 & $\mathrm{BH} 88$ & 1569 & 12461 & 11868 & 12,6 & 13,2 & 95,2 \\
\hline Longvic 3 & $\mathrm{BH} 88$ & 1105 & 23889 & 19794 & 4,6 & 5,6 & 82,9 \\
\hline Lux 1 & $\mathrm{BH} 88$ & 36 & 6620 & 5268 & 0,5 & 0,7 & 79,6 \\
\hline Norges 1 & $\mathrm{BH} 88$ & 230 & 9211 & 6913 & 2,5 & 3,3 & 75,1 \\
\hline Pralon 1 & $\mathrm{BH} 88$ & 279 & 4682 & 3590 & 6,0 & 7,8 & 76,7 \\
\hline Saussy 1 & $\mathrm{BH} 88$ & 351 & 6640 & 2591 & 5,3 & 13,5 & 39,0 \\
\hline Val Suzon 1 & $\mathrm{BH} 88$ & 120 & 6893 & 2864 & 1,7 & 4,2 & 41,5 \\
\hline Total 1988 & & 4480 & 91262 & 69910 & 4,9 & 6,4 & 76,6 \\
\hline Auvillars 1 & $\mathrm{BH} 90$ & 276 & 10629 & 9923 & 2,6 & 2,8 & 93,4 \\
\hline Bretenières 1 & $\mathrm{BH} 90$ & 44 & 673 & 500 & 6,5 & 8,8 & 74,3 \\
\hline Bretenières 2 & $\mathrm{BH} 90$ & 32 & 1272 & 759 & 2,5 & 4,2 & 59,7 \\
\hline Fleuret 1 & $\mathrm{OH} 90$ & 52 & 5088 & 3270 & 1,0 & 1,6 & 64,3 \\
\hline Mailly 1 & $\mathrm{BH} 90$ & 218 & 12274 & 10643 & 1,8 & 2,0 & 86,7 \\
\hline Marliens 1 & $\mathrm{BH} 90$ & 249 & 13820 & 8645 & 1,8 & 2,9 & 62,6 \\
\hline Pasques 1 & $\mathrm{BH} 90$ & 493 & 5235 & 3057 & 9,4 & 16,1 & 58,4 \\
\hline Remilly 1 & $\mathrm{BH} 90$ & 42 & 1645 & 1166 & 2,6 & 3,6 & 70,9 \\
\hline Remilly 2 & $\mathrm{BH} 90$ & 39 & 1185 & 992 & 3,3 & 3,9 & 83,7 \\
\hline Rouvres 1 & $\mathrm{BH} 90$ & 44 & 1006 & 659 & 4,4 & 6,7 & 65,6 \\
\hline Total 1990 & & 1489 & 52827 & 39614 & 2,8 & 3,8 & 75,0 \\
\hline Total 1986-1990 & & 8602 & 205288 & 155218 & 4,2 & 5,5 & 75,6 \\
\hline
\end{tabular}

BH : blé tendre d'hiver ; OH : orge d'hiver ; CO : colza d'automne ; Cult/ann : culture et année de semis ; Lev : levées au champ ; DT : dénombrement réel total ; DV : dénombrement réel viable ; L/DT : rapport calculé levées/dénombrement total ; LDV : rapport calculé levées/dénombrement viable ; DV/DT : viabilité moyenne. 
stock semencier viable et $4,2 \%$ du stock semencier total, et $75 \%$ des taux parcellaires de levée sont compris entre 13,8 et $1,8 \%$ pour le stock viable et entre 9,4 et $1,5 \%$ pour le stock total (tableau I).

Le taux annuel moyen de levée est plus élevé au cours de la campagne 1986/1987 qu'au cours des campagnes suivantes, et ceci est vrai aussi pour les trois espèces les mieux représentées (Alopecurus myosuroides, Anagallis arvensis et Viola arvensis). Ces différences pourraient trouver un début d'explication dans la comparaison des données climatiques (fig 1-6) : pendant la période de formation des semences, l'année $1985 / 1986$ fut nettement plus sèche que les autres, avec des températures moyennes comparables, ce qui a pu entraîner une moindre dormance des semences à la dissémination, mais surtout l'hiver 1986/1987 fut plus précoce, plus long, plus froid ou plus régulier que les autres, ce qui a pu entraîner une plus complète stratification permettant une meilleure levée de dormance des semences enfouies.

\section{Relations stock-levées spécifiques}

Pour calculer les taux spécifiques de levée, nous n'avons retenu que les parcelles dans lesquelles l'espèce était présente avec un stock semencier mesuré supérieur à 100 semences par mètre carré.

Le tableau II présente, pour les espèces les mieux représentées sur l'ensemble des 35 parcelles, les taux spécifiques médians de levée, et pour les espèces ayant été observées sur plus de dix parcelles, les valeurs extrêmes des taux spécifiques de levée comprenant $80 \%$ des valeurs observées. Ainsi par exemple pour Alopecurus myosuroides, le taux médian de levée par rapport au dénombrement total est égal à $8,5 \%$ et $80 \%$ des taux mesurés par parcelle sont compris entre 23,7 et 4,5 . Cette présentation permet de donner une idée plus raisonnable de la variabilité dans l'espace et dans le temps du taux spécifique de levée.

En complément, pour quelques espèces moins bien représentées, le taux moyen de levée, préférable au taux médian compte tenu du faible nombre d'observations (trois ou quatre), était de 5,3 et $6,5 \%$ (par rapport aux stocks total et viable respectivement) pour Fallopia convolvulus Löve, 10,8 et $31,6 \%$ pour Galium aparine L, 4,1 et $4,1 \%$ pour Sonchus asper Hill et 13,4 et $19,1 \%$ pour Veronica hederifolia $L$.
Par rapport tant au dénombrement total qu'au dénombrement viable, le taux médian de levée est toujours faible; il ne dépasse $5 \%$ que pour les espèces dont les semences peuvent lever même lorsqu'elles sont enfouies à des profondeurs supérieures à $5 \mathrm{~cm}$ : c'est le cas d'Alopecurus myosuroides, Fallopia convolvulus, Galium aparine, Polygonum persicaria, Veronica hederifolia (Chancellor, 1964 ; Barralis et al, 1988).

\section{Viabilité moyenne des semences}

Le taux de viabilité, mesuré par le nombre de semences aptes à germer par rapport au nombre total de semences dénombrées, est très variable entre espèces, allant de $98 \%$ chez Matricaria perforata ou Veronica persica à $20 \%$ chez Polygonum persicaria (tableau II).

Les espèces dont le taux moyen de viabilité est supérieur à 80 ou inférieur à $20 \%$ montrent une très grande stabilité spatiotemporelle de ce taux (pour ces espèces, le coefficient de corrélation entre dénombrement viable et dénombrement total est supérieur à 0,95 ). En revanche les espèces dont le taux moyen est compris entre 80 et $20 \%$ montrent une très forte variabilité (le coefficient de corrélation est compris entre 0,90 et 0,60 ) qui peut être la conséquence d'une mortalité in situ des semences sans altération de leur morphologie (Roberts, 1981), ou plus vraisemblablement d'une dormance induite importante que le traitement de levée de dormance mis en œuvre dans notre protocole ne permet pas de lever totalement dans certaines situations.

\section{Évolution de la flore levée}

La présence des mauvaises herbes à une période définie de l'année, en particulier en fin d'automne et au début du printemps est importante à connaître pour judicieusement choisir les traitements herbicides les plus efficaces et les mieux adaptés à la flore réellement présente.

Le tableau III présente la densité relative des levées à ces deux dates en fonction de la somme des levées observées au cours du cycle cultural, et permet de classer les espèces en trois groupes : levée automnale préférentielle (Alopecurus myosuroides, Capsella bursa pastoris), levée automnale à printanière plus ou moins prolongée (Anagallis arvensis, Aphanes arvensis, Chenopodium album (dont les levées autom- 

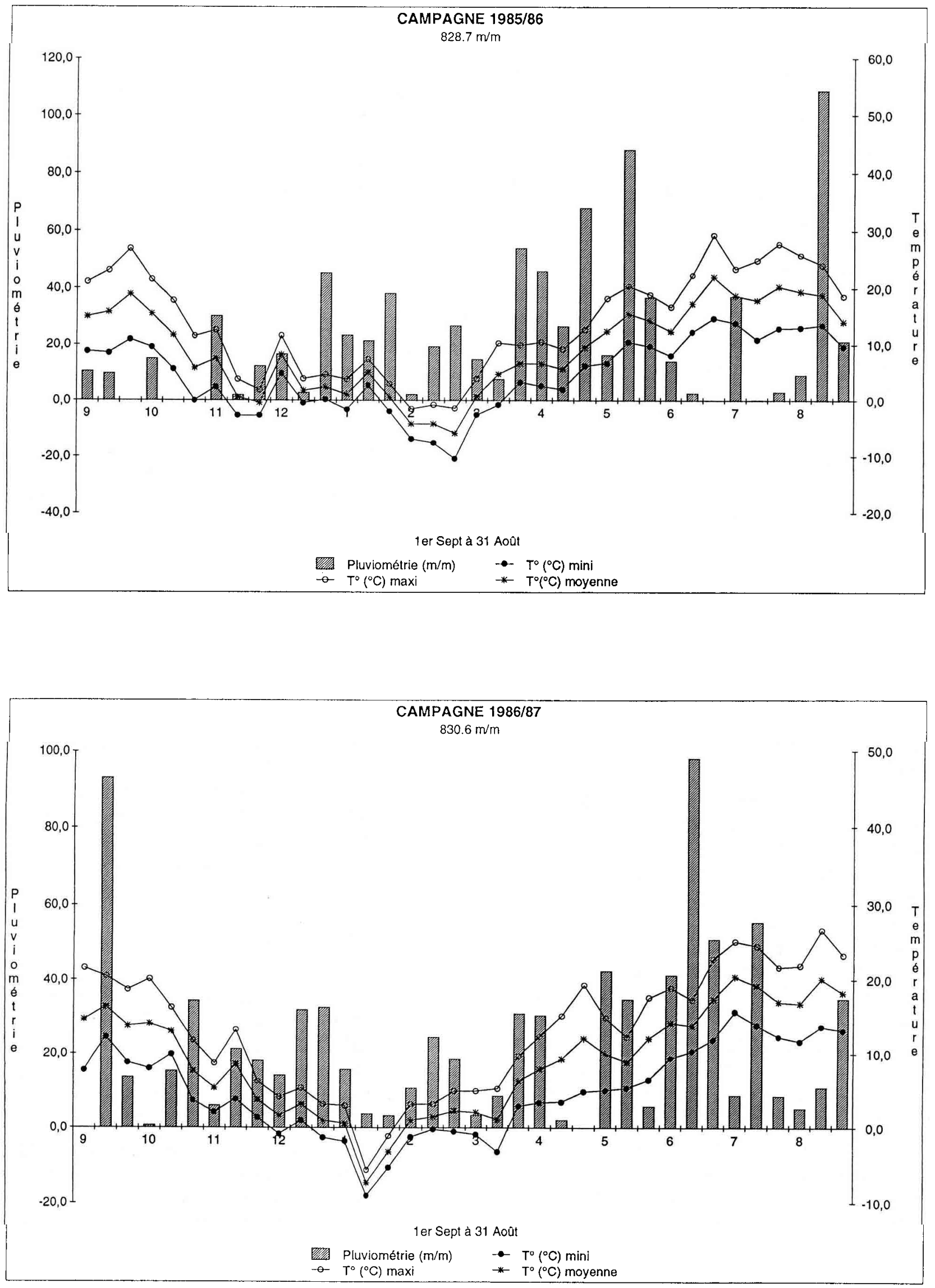

Figs 1-6. Données climatiques de la station météorologique du domaine d'Époisses (21). 

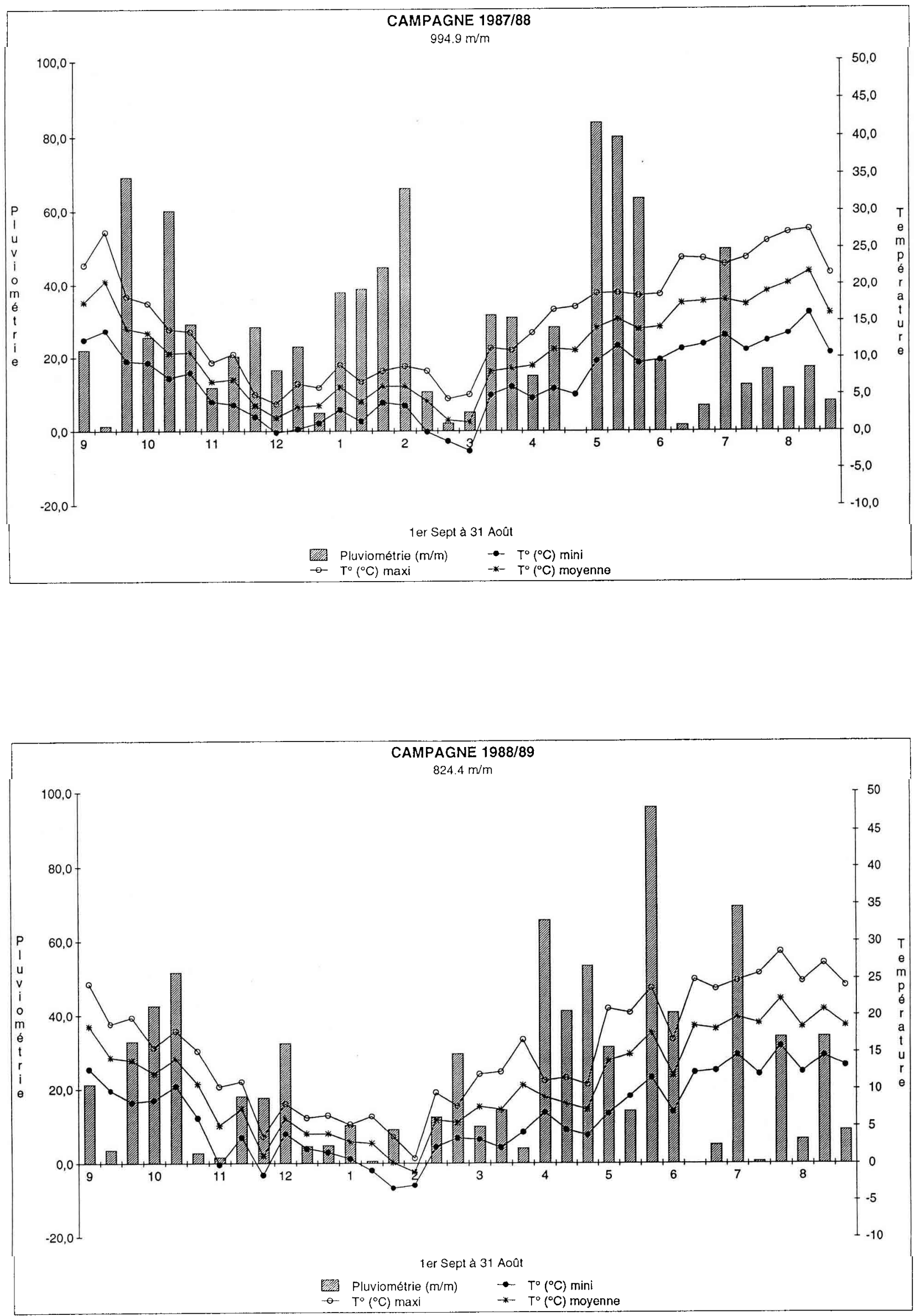

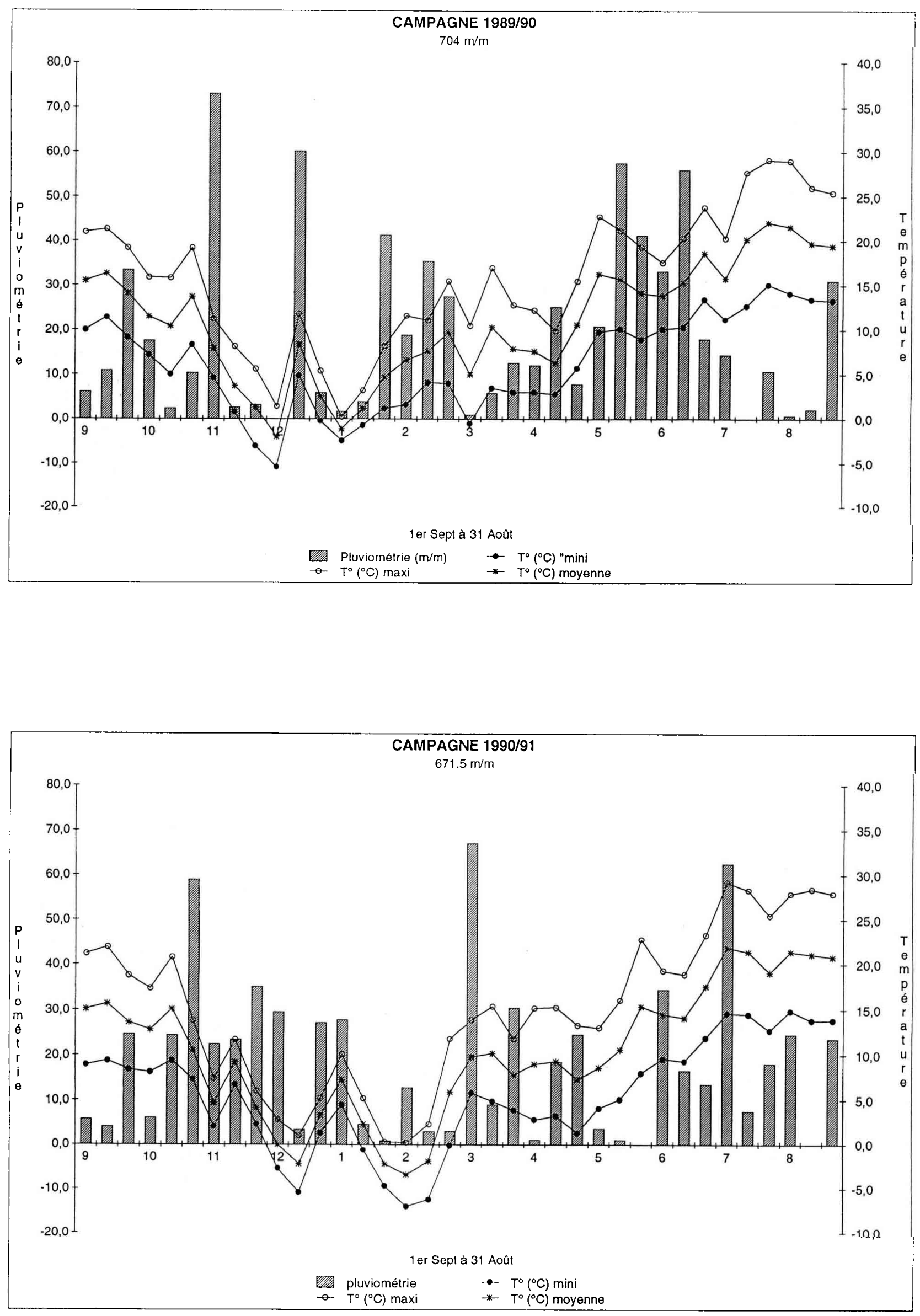
Tableau II. Taux médians de levée et taux extrêmes (pour les espèces présentes dans plus de 10 parcelles) en fonction du dénombrement total (L/DT) ou viable (L/DV), et viabilité moyenne des semences (DV/DT).

\begin{tabular}{|c|c|c|c|c|c|c|}
\hline & \multirow[t]{2}{*}{ Nombre a } & \multicolumn{2}{|c|}{ Médianes } & \multicolumn{2}{|c|}{ Extrêmes } & \multirow{2}{*}{$\begin{array}{l}\text { Viabilité } \\
\text { DVIDT }\end{array}$} \\
\hline & & $L / D T$ & L/DV & $L D T$ & LIDV & \\
\hline Aethusa cynapium $\mathrm{L}$ & 5 & 3,6 & 3,6 & & & 94,8 \\
\hline Alopecurus myosuroides Huds & 22 & 8,5 & 16,5 & $23,7-4,5$ & $31,5-4,5$ & 85,4 \\
\hline Anagallis arvensis $\mathrm{L}$ & 20 & 2,8 & 5,1 & $6,6-1,3$ & $17,5-1,7$ & 48,8 \\
\hline Aphanes arvensis $\mathrm{L}$ & 5 & 2,9 & 3,1 & & & 76,9 \\
\hline Capsella bursa pastoris Med & 11 & 1,6 & 1,6 & $2,6-0,6$ & $2,6-0,3$ & 97,5 \\
\hline Chenopodium album $\mathrm{L}$ & 12 & 1,8 & 2,8 & $6,8-0,4$ & $14,6-0,7$ & 69,8 \\
\hline Euphorbia exigua L & 9 & 0,8 & 1,4 & & & 49,3 \\
\hline Kickxia spuria Dumort & 6 & 0,5 & 0,9 & & & 70,1 \\
\hline Matricaria perforata Merat & 5 & 3,8 & 3,8 & & & 98,6 \\
\hline Papaver rhoeas $\mathrm{L}$ & 8 & 2,3 & 2,7 & & & 74,3 \\
\hline Polygonum persicaria $\mathrm{L}$ & 5 & 7,0 & 38,2 & & & 20,2 \\
\hline Veronica persica Poiret & 12 & 3,3 & 3,3 & $11,5-0,7$ & $11,5-0,7$ & 97,9 \\
\hline Viola arvensis Murray & 15 & 2,7 & 3,2 & $9,9-0,7$ & $15,3-1,1$ & 51,2 \\
\hline
\end{tabular}

${ }^{a}$ Nombre de parcelles où le stock semencier est supérieur à 100 semences par mètre carré.

nales disparaissent en hiver), Matricaria perforata, Papaver rhoeas, Veronica hederifolia, $V$ persica, Viola arvensis), levée printanière à estivale (Aethusa cynapium, Euphorbia exigua, Kickxia spuria, Polygonum persicaria).

Il est intéressant de noter qu'au cours de la campagne 1986/1987 dont l'hiver fut le plus rigoureux les levées ont été relativement moins importantes à l'automne et plus importantes au printemps ; nous l'avions observé chez Alopecurus myosuroides (Barralis et Chadœuf,
1988) et nous retrouvons cette tendance chez Viola arvensis. Au cours de cette campagne où le taux annuel moyen de levée a été le plus fort, les levées automnales ont représenté respectivement pour ces deux espèces 32,4 et $48,8 \%$ alors que pour les trois autres campagnes elles ont représenté 77,7 et $58,9 \%$ du total des levées. Cet effet année ne se retrouve pas chez Anagallis arvensis dont les levées sont plus tardives avec un taux moyen de levée automnale de $19,7 \%$.

Tableau III. Taux de levée cumulée au champ (sur l'ensemble des parcelles) au 1er décembre (L12) et au $1^{\text {er }}$ avril (LO4) en fonction du nombre total de levées observées pendant le cycle végétatif de la culture.

$L 12 \quad L O 4$

Aethusa cynapium

0,0

72,0

Alopecurus myosuroides

Anagallis arvensis

Aphanes arvensis

Capsella bursa pastoris

Chenopodium album

Euphorbia exigua

Kickxia spuria

Matricaria perforata

Papaver rhoeas

Polygonum persicaria

Veronica hederifolia

Veronica persica

Viola arvensis
19,7

44,6

69,6

18,4

0,0

0,0

47,1

52,9

0,0

60,2

54,8

57,1
43,8

98,5

60,2

78,4

89,2

74,3

37,5

30,0

92,5

97,1

65,1

100,0

92,0

92,2 


\section{DISCUSSION ET CONCLUSIONS}

La levée au champ est une étape importante du cycle de développement d'une mauvaise herbe annuelle, et la connaissance des relations entre le stock semencier et la densité des levées est un élément majeur pour la prévision des infestations et la définition de stratégies raisonnées de désherbage. Elle est considérée comme un paramètre critique pour tout modèle de gestion des mauvaises herbes (Forcella et al, 1993).

Cette étude confirme qu'il existe une relation entre stock et levées, mais la densité des levées dans une culture ne représente qu'une faible proportion du nombre de semences enfouies dans la couche arable. Le nombre de levées, mesuré sur 35 parcelles au cours de quatre campagnes, ne représente que $5,5 \%$ du nombre total de semences viables; c'est une valeur qui confirme plusieurs travaux antérieurs pour des populations plurispécifiques : moyenne pour sept parcelles semées en blé d'hiver 5,6 \% (Barralis, 1972), moyenne pour cinq parcelles maraîchères $5,9 \%$ (Carretero, 1977), moyenne pour deux parcelles de blé d'hiver 3,3\% (Beuret, 1984), moyenne pour deux parcelles en céréales d'hiver 3,6\% (Debaeke, 1988b).

Le tableau IV présente, pour quelques espèces importantes, les résultats acquis dans des expérimentations similaires. Pour ces espèces à germination indifférente ou plutôt printanière (Montégut, 1975) les différences sont dans l'ensemble assez peu marquées. De même pour Alopecurus myosuroides, espèce à germination plutôt automnale, le taux de levée est estimé dans les céréales d'hiver à 18,0 \% (Moss, 1990) contre 16,5 dans notre expérimentation.

Cette relation entre le stock semencier et les levées au champ peut varier en fonction des conditions climatiques (en particulier de la tem- pérature et de l'humidité du sol), mais elle varie aussi en fonction des caractéristiques physiques du sol (finesse et tassement) qui modifient le comportement des semences et la levée au champ (Roberts et Hewson, 1971), et surtout en fonction des caractéristiques culturales. L'effet du travail du sol sur le mouvement et l'enfouissement des semences (Moss, 1988 ; Dessaint et al, 1996a) est bien connu, et le taux de levée est d'autant plus faible que les semences sont profondément enfouies (Roberts et Feast, 1972 ; Froud-Williams et al, 1984). La date de semis ou de la dernière façon culturale avant semis (Roberts et Ricketts, 1979 ; Debaeke, 1988a ; Beuret, 1989) ainsi que le nombre de façons culturales après semis (Roberts et Feast, 1972) ont aussi un effet sur la densité des levées.

C'est la raison pour laquelle les taux spécifiques de levée que nous avons mesurés dans les cultures d'automne après labour seraient certainement différents, particulièrement pour les espèces à périodicité de germination bien marquée, espèces à germination assez strictement hivernale ou estivale, s'ils avaient été mesurés dans des cultures de printemps ou après travail superficiel et ne sont donc pas transposables à ces conditions-ci.

Les différences que l'on peut observer entre auteurs ont en fait trois origines possibles : i) la variabilité des conditions environnementales, édaphoclimatiques et phytotechniques, ii) la variabilité infraspécifique qui peut expliquer le comportement variable des espèces vis-à-vis du milieu, iii) les méthodologies analytiques. De ce dernier point de vue, il faut particulièrement insister sur la variabilité des mesures : pour le stock semencier, le nombre, le diamètre et la hauteur des prélèvements parcellaires, et pour les levées, le dénombrement fréquent et continu ou le dénombrement ponctuel à quelques périodes seulement du cycle cultural.

Tableau IV. Comparaison du taux de levée de quelques espèces.

Blé d'hiver ${ }^{\mathrm{a}}$

Anagallis arvensis

Capsella bursa pastoris

Chenopodium album

Euphorbia exigua

Papaver rhoeas

Viola arvensis
5,1

1,6

2,8

1,4

2,7

3,2
Blé d'hiver ${ }^{\mathrm{b}}$

Orge de printemps c

a Nos résultats ; ${ }^{b}$ Debaecke (1988b) ; c Wilson et Aebischer (1995).

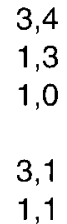

1,1 
C'est pourquoi les méthodologies à mettre en œuvre doivent permettre d'estimer le stock avec une précision suffisante (Dessaint et al, 1996b) et les levées tout au long du cycle cultural afin de dénombrer la totalité des levées, ainsi que la période de levée des différentes cohortes, et non seulement la densité des levées à un moment donné.

Enfin la chronologie des levées au champ peut être modifiée par les conditions climatiques de l'hiver, particulièrement pour les espèces à germination automnale stricte ou préférentielle.

Le paramétrage du cycle de développement d'une mauvaise herbe nécessite des recherches complémentaires et spécifiques sur la densité du stock semencier, le taux et la dynamique des levées, la productivité semencière (Forcella et al, 1993). Complétée par l'analyse des interactions entre plante cultivée et mauvaises herbes, la connaissance et la modélisation des risques d'envahissement des cultures est à la base de toute stratégie raisonnée de désherbage dans la rotation.

\section{REMERCIEMENTS}

Les auteurs remercient $P$ Girard qui a participé à l'analyse floristique des parcelles ainsi que les agriculteurs qui nous ont permis de réaliser ce travail.

\section{RÉFÉRENCES}

Ball DA, Miller SD (1989) A comparison of techniques for estimation of arable soil seedbanks and their relationship to weed flora. Weed Res $29,365-373$

Barralis G (1972) Évolution comparative de la flore adventice avec ou sans désherbage chimique. Weed Res 12, 115-127

Barralis G, Chadœuf R (1980) Étude de la dynamique d'une communauté adventice. I. Évolution de la flore adventice au cours du cycle végétatif d'une culture. Weed Res 20, 231-237

Barralis G, Chadœuf R (1987) Potentiel semencier des terres arables. Weed Res 27, 417-424

Barralis G, Chadœuf R (1988) Relations entre flore potentielle et flore réelle des champs cultivés. VII/e Coll Intern Biol Écol Syst Mauvaises Herbes 43-52

Barralis G, Chadœuf R, Lonchamp JP (1988) Longévité des semences de mauvaises herbes annuelles dans un sol cultivé. Weed Res 28, 407-418

Barralis G, Salin D (1973) Relations entre flore potentielle et flore réelle dans quelques types de sols de Côte-d'Or. IVe Coll Intern Écol Biol Mauvaises Herbes 94-101
Beuret E (1984) Stocks grainiers des sols et pratiques culturales : la relation flore potentielle-flore réelle. Schweiz Landw Forsch 23, 89-96

Beuret E (1989) Influence des pratiques culturales sur l'évolution de la flore adventice : étude du potentiel semencier des sols. Revue Suisse Agric 21, 75-82

Chancellor RJ (1964) The depth of weed seed germination in field. 7th British Weed Control Conf 607-613

Carretero JL (1977) Estimacion del contenido de semillas de malas hierbas de un solo agricola como prediccion de su flora adventicia. Anal Inst Bot Cavanilles 34, 267-278

Debaeke P (1988a) Modélisation de l'évolution à long terme de la flore adventice. II. Application à trois dicotylédones annuelles en un site donné. agronomie 8, 767-777

Debaeke P (1988b) Dynamique de quelques dicotylédones adventices en culture de céréale. I. Relation flore levée-stock semencier. Weed Res 28, 251-263

Dessaint F, Chadœuf R, Barralis G (1966a) Influence of tillage on the vertical distribution of simulated plastic seeds. Sec Intern Weed Control Congress 95-100

Dessaint F, Barralis G, Caixinhas ML, Mayor JP, Recasens J, Zanin G (1996b) Precision of soil seedbank sampling: how many soil cores. Weed Res 36, 143-151

Forcella F, Wilson RG, Renner KA, Dekker, J, Harvey RG, Alm DA, Buhler DD, Cardina J (1992) Weed seedbanks of the US corn belt: magnitude, variation, emergence and application. Weed Science 40 , 636-644

Forcella F, Eradat-Oskoui K, Wagner ST (1993) Application of weed seedbank ecology to low-input crop management. Ecological Applications 3, 74-83

Froud-Williams RJ, Chancellor RJ, Drennan (1984) The effects of seed burial and soil disturbance on emergence and survival of arable weeds in relation to minimal cultivation. J App/ Ecol 21, 629-641

Jensen HA (1969) Content of buried seeds in arable soil in Denmark and its relation to the weed population. Dansk Bot Ark 27, 1-56

Kropac Z (1966) Estimation of weed seeds in arable soil. Pedobiologia 6, 105-128

Lambelet-Haueter C (1986) Analyse de la flore potentielle, en relation avec la flore réelle, en grandes cultures de la région genevoise. Candollea 41, 299323

Montegut J (1975) Écologie de la germination des mauvaises herbes. In : La germination des semences ( $R$ Chaussat, $Y$ Le Deunff, eds), Paris, Gauthier-Villars, 191-217

Moss RS (1988) Influence of cultivations on the vertical distribution of weed seeds in the soil. VIIIe Coll Intern Biol Ecol Syst Mauvaises Herbes, 71-80

Moss RS (1990) The seed cycle of Alopecurus myosuroides in winter cereals: a quantitative analysis. Proc EWRS Symposium, Integrated Weed Management in Cereals, 27-36 
Roberts HA (1981) Seed banks in soil. Adv App/ Biol6, 1-55

Roberts HA (1984) Crop and weed emergence patterns in relation to time of cultivation and rainfall. Ann App/ Biol 105, 263-275

Roberts HA, Chancellor RJ (1986) Seed banks of some arable soils in the English midlands. Weed Res 26, 251-257

Roberts HA, Feast PM (1972) Fate of seeds of some annual weeds in different depths of cultivated and undisturbed soil. Weed Res 12, 316-324

Roberts HA, Hewson RT (1971) Herbicide performance and soil surface conditions. Weed Res 11, 69-73
Roberts HA, Ricketts ME (1979) Quantitative relationships between the weed flora after cultivation and the seed population in the soil. Weed Res 19, 269-275

Wilson BJ, Lawson HM (1992) Seedbank persistence and seedling emergence of seven weed species in autumn-sown crops following a single year's seedling. Ann App/ Biol 120, 105-116

Wilson PJ, Aebischer NJ (1995) The distribution of dicotyledonous arable weeds in relation to distance from field edge. J Appl Ecol 32, 292-310

Zanin G, Mosca G, Catizone P (1992) A profile of the potential flora in maize fields of the Po valley. Weed Res 32, 407-418 